\title{
Automatic Street Light Control System
}

\author{
Badri Narayan Sahoo, Jeeban Jyoti Mahakud, Priyabrata Pattanaik
}

\begin{abstract}
This paper is concerned with the automation in street lighting system. The projected work describes the automatic working of street lights according to seasons and timing of a day. The system comprises of microcontroller, GPS, RTC to implement the work. Projected work overcomes the drawbacks of previous systems like is error in timing, by using the information about time with the help of GPS. Microcontroller takes the data and accordingly operation of lighting on/off is performed. RTC comes into action in case of any interruption. The system works without any operator. The developed have some advantages like energy saving as lights are on according to timings and increase in life of luminaire.
\end{abstract}

Index Terms-Microcontroller, Microcontroller, GPS, streetlights, RTC, UART

\section{INTRODUCTION}

Now-a-days, street lights consume large part of power. Because there is no automation in this field, there always require a human being to turn on/off the light according to the need of light. If the person that is employed for this work is absent then this would result in large amount of wastage of light energy. So, there is a need of an automatic system that would help in conservation of electrical energy. A system that automatically controls the on/off function of light according requirement is needed [1].

There are some cases in which street lights have pre-set time for turning on/off light and if due to some reason timer is not working in a proper way then it would take time to fix it because it has long procedure of complaining and then working on it to resolve the problem. The environment is also affected if the light remains in working condition (on condition) for a long time[2].

Currently LEDs (Light emitting diodes) are used that provide more luminance and less power consumption as compared to previous ones like halogen lamps, mercury lamps etc. The street lights operation is based on light sensor which makes them separate from other entities[3].

To on/off street lights controllers have a real time clocks IC (RTC) which is not that accurate and the error of time is plus or minus 5 seconds a day, so it involve resetting in a month[4][5]. Another disadvantage of them is dependency on battery system for uninterrupted power supplement.

If the battery is not working in a proper manner or gets damaged then the whole system requires resetting.

Revised Manuscript Received on September 10, 2019.

Badri Narayan Sahoo, Dept. of Electronics \& Communication Engineering, Siksha $\mathrm{O}$ Anusandhan Deemed to be University, Odisha, India.

(Email: badrinarayansahu@soa.ac.in)

Jeeban Jyoti Mahakud, Dept. of Electronics \& Communication Engineering, Siksha O Anusandhan Deemed to be University, Odisha, India.

(Email: jeevanmahakud@soa.ac.in)

Priyabrata Pattanaik, Dept. of Electronics \& Communication Engineering, Siksha O Anusandhan Deemed to be University, Odisha, India.

(Email: priyabratapattanaik@soa.ac.in)
So, for the energy conservation we require a new technology which overcomes the above mentioned difficulties and reduces as well as energy wastage.

The main objective of our invention is to implement a lighting control system that provides sufficient control by using the GPS in the system.

There exist many systems that provides control and monitoring over lights such as radio channel control system and supply change system etc. However, these systems have some disadvantages like in radio channel control system creates the problem of pollution as it uses radio waves and it is not cost efficient. The other system has a disadvantage of cost of autotransformer and we need to put a microcontroller in each and every street to provide control over lights. To overcome these problems we have projected a system for controlling lighting of street with the help of GPS[6].

Second section of the paper discusses about development of the system for controlling street lighting in which different types are modules are discussed. Third section tells the results that we have got from our experiment. Then the lastly we have concluded the experimentation by following the work done.

\section{DEVELOPMENT OF PROJECTED SYSTEM}

A 'GPS' is employed in the system to find the real local time by the data of 'GPS'. The time of sun rise and sun set agreeing to its geographical is decided. After the determination of time the controlling of street light can be achieved depending on the time.

The projected work overcomes the difficulties of previous existing systems, it finds the geographical location, date and time by microcontroller and GPS. It also provides the data about present situation of the system. The microcontroller takes the time and then controls the lights according to requirement (on/off). By the projected system the error of time is very small it's about hundred nanoseconds which is the time for transmission of data to the receiver.

During the interruption of power supply power the operation is performed by an "RTC" and the time to reconnect the GPS satellite to the system depend on the location and generally it is in the range of (15secs3 minutes).

The data always gets updated by a software provided within the "RTC' that helps in coring the error during the reconnection of GPS satellite to the system. The reliability of the system is high and it comprises of 2 modules, they can work together or independently. First module is to provide on/off operation of light, the module is put in the control panel of the main system of street light. Second module is to control the luminance of lights and it is present in each and every street.

Published By: 
The main thing which we are using in the system is GPS and it's a real time system. The time to on or off the lights depend on the data received by the GPS.

1) First module :

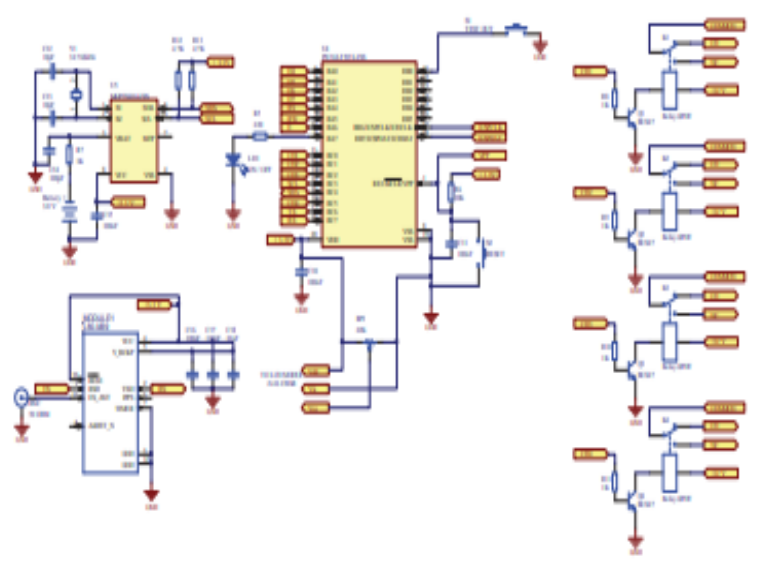

Figure 1 Schematic Presentation for First Module

The first module is which is placed in the dashboard consists inherent antenna to obtain data by the GPS. A connector is employed on the sites where the GPS signal is not good to link external active and passive antenna. Figure 1 shows the representation of first module. In this system there is no need of operator to for adjustment of the system and for operating lights. This feature makes the system error free. In the presence of day light the system makes the luminaire in off condition and after sunset switch on the street lights.

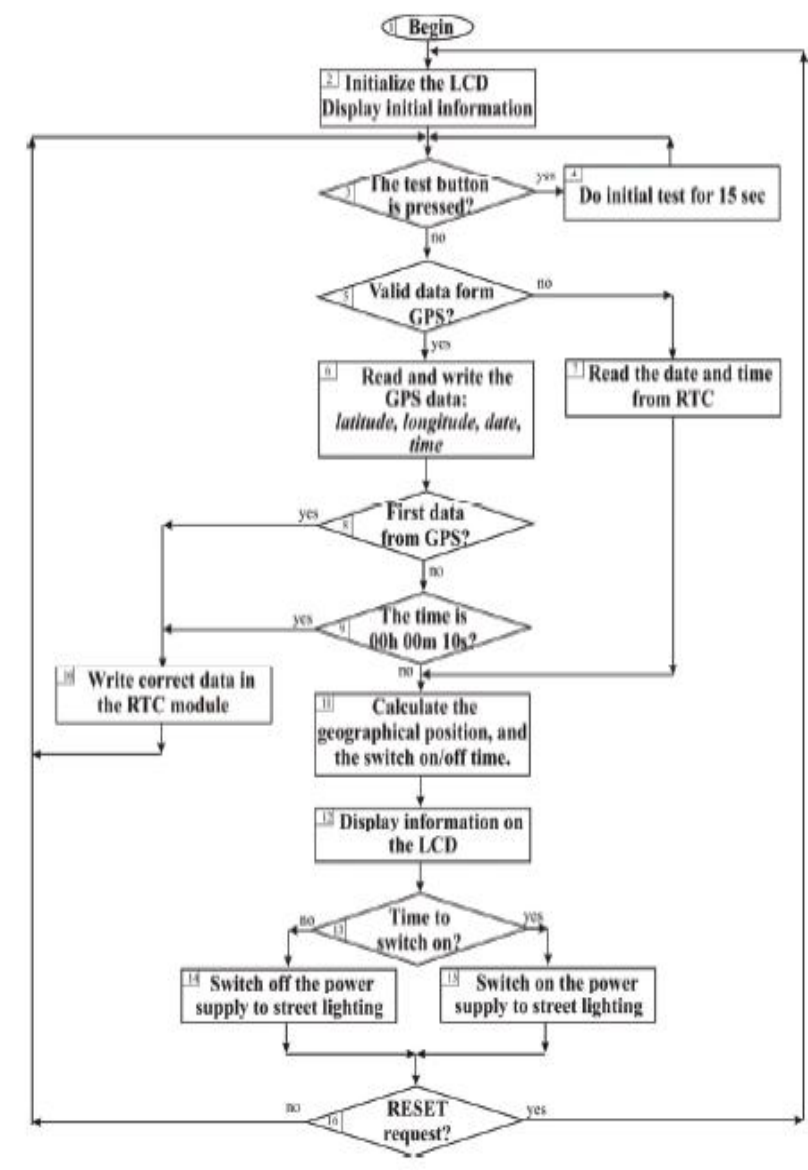

Figure 2 Algorithm for First Module
Figure 2 is a flow chart of algorithm wherein there are sixteen blocks.

\section{Initial step:}

In first step LCD is initialized and initial information is displayed by the LCD display.

\section{Second step:}

In second step it is checked if the test button is presses or not, if yes then the initial test would start for $15 \mathrm{~min}$. and then go to the previous step again. If the button is yet not pressed then it move further to the next step and the next step is,

\section{Third Step:}

Checking of GPS data, if it is valid then move further and if not extraction of date and time is done by RTC

\section{Fourth Step:}

Read and write the GPS data. After this,

\section{Fifth Step:}

is to check whether this is first data from GPS or not. If yes the procedure would jump to the tenth block otherwise to the next step which is,

\section{Sixth Step:}

is to check whether the actual time $00 \mathrm{~h} 00 \mathrm{~m} 10 \mathrm{sec}$. If yes, RTC module is updated, and if not

\section{Seventh Step:}

Calculation of geographical position and the operation on/off for lights.

\section{Eighth Step:}

Displaying information on LCD;

\section{Ninth Step:}

is to check the timing to switch on the lights or not. If yes, then switch on the power supply otherwise switch off the supply power. And then reset the program.

The calculations for block eleven is given below:

- Calculation of the fractional year $\mathrm{G}$ in degrees:

$$
G=\frac{360}{365.25} *\left(N+\frac{T}{24}\right)
$$


where:

$\mathrm{N}=$ day number of teh year. For example January $1=$ day 1, January $2=$ day 2 , etc.

$\mathrm{T}$ - is the local time and it is expressed in fractions of

$\mathrm{T}=$ hour + minutes $/ 60$

- Calculation of the time correction for solar angle TC: $\mathrm{TC}=0.004297+0.107029 * \cos (\mathrm{G})-1.837877 * \sin (\mathrm{G})$ $0.837378 * \cos (2 * \mathrm{G})-2.340475 * \sin (2 * \mathrm{G})$

- Calculation of the declination of the sun D: $\mathrm{D}=0.396372-22.91327 * \cos (\mathrm{G})+4.02543 * \sin (\mathrm{G})-$ $0.387205 * \cos (2 * \mathrm{G})+0.051967 * \sin (2 * \mathrm{G})-0.154527 * \cos (3 * \mathrm{G})+$ $0.084798 * \sin (3 * \mathrm{G})$

- Calculation of the the Solar Hour Angle SHA: $\mathrm{SHA}=(\mathrm{T}-12)^{*} \cdot 15+$ Longitude $+\mathrm{TC}$

Longitude in degrees (this figure should be negative Weast of Greenwich and positive East of Greenwich).

- Calculation of the Sun Zenith Angle SZA:

$$
\cos (\mathrm{SZA})=
$$

$\sin ($ Latitude $) * \sin (\mathrm{D})+\cos ($ Latitude $) * \cos (\mathrm{D}) * \cos (\mathrm{SHA})$

$$
\mathrm{SZA}=
$$

$\arccos (\sin ($ Latitude $) * \sin (\mathrm{D})+\cos ($ Latitude $) * \cos (\mathrm{D}) * \cos (\mathrm{SHA}))$

There are some related terms:

Civil twilight, Navigation twilight, and astronomical twilight.

\section{Navigation twilight:}

"finishes in the evening once the center of the solar disk touches $12^{\circ}$ under the horizon about this moment, human visualisation is tough to differentiate the horizon itself".

\section{Civil twilight:}

"initiates at the sunset on an even horizon and finishes once the center of the solar disk slopes 6 degrees under the horizon. About the finish of the Civil Twilight, outside work and the difference of colours of usual light are already hindered, and the sunniest stars and planets can be seen across the sky". It is suggested to switch on street lights.

\section{The astronomical twilight:}

finishes at the sunset when the center of the solar disk extents eighteen degrees underneath the horizon.

According to studies in the close of Civil Twilight, the system switches.

\section{2) Second Module:}

There exists an output which is pulse width modulated output in the second module which is placed in each and every luminaire of the street. The voltage range of the supply voltage is in-between $5 \mathrm{~V}$ to $37 \mathrm{~V}$. The GPS data is also accessed by the second module to check the weather condition if it is summer or winter season according to which no. of luminaries are planned and their intensity is adjusted.

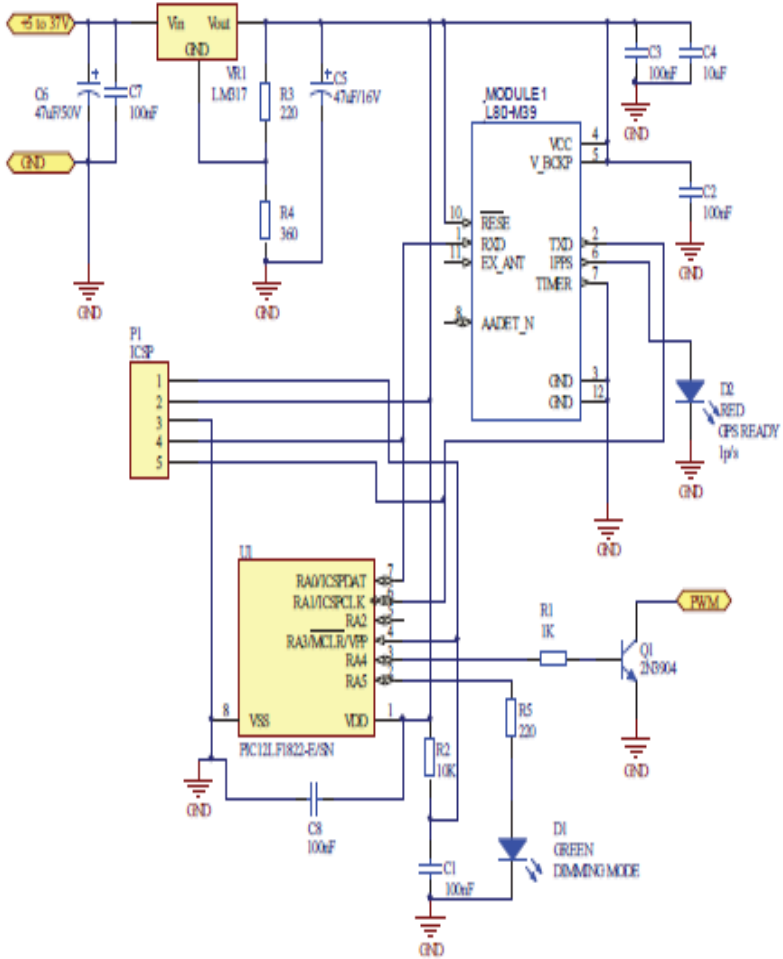

Figure 3 Schematic Representation of Second Module

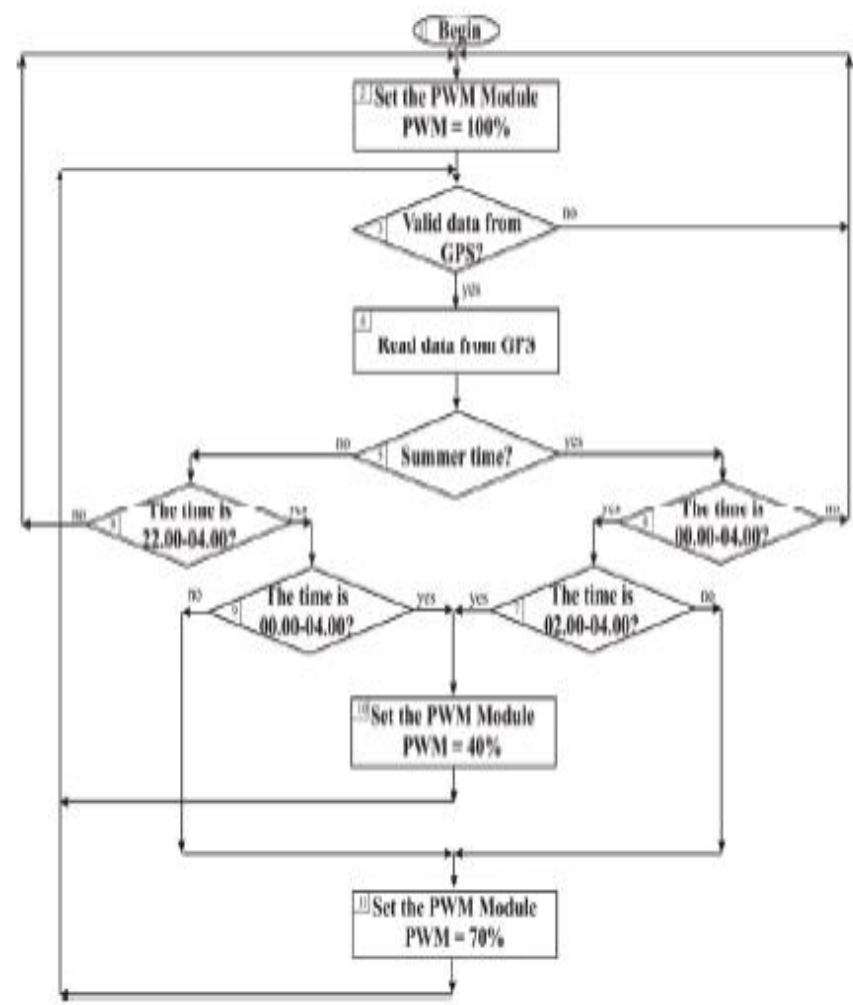

Figure 4 Flow chart of algorithm of second module

As we can see from the flow chart in starting of the algorithm PWM setting which ensures the lighting even in the case of interruption in the system is set to 100 percentage. Then GPS interacted by the device and check whether the data from GPS is effective or not. If not, the algorithm goes to step 1, the setting of lights is set to 100 
percent otherwise it would read data from GPS. Then further checks the weather condition and the seasons are winter and summer season.

' 3 ' stages for lowering the intensity of light are delivered for every period shown in table.

Table I: ' 3 ' stages for lowering the intensity of light

\begin{tabular}{|c|c|c|c|}
\hline $\begin{array}{l}\text { Timing for } \\
\text { summers }\end{array}$ & $\begin{array}{l}\text { \% age } \\
\text { setting for } \\
\text { summers }\end{array}$ & $\begin{array}{l}\text { Timing for } \\
\text { winters }\end{array}$ & $\begin{array}{l}\% \text { age for } \\
\text { winters }\end{array}$ \\
\hline $\begin{array}{l}\text { from lighting } \\
\text { up } \\
\text { 00:00:00 }\end{array}$ & $100 \%$ & $\begin{array}{l}\text { From } \\
\text { lighting up } \\
\text { to 22:00:01 }\end{array}$ & $100 \%$ \\
\hline $\begin{array}{l}\text { From } \\
\text { 00:00:00h. } \\
\text { Until } \\
\text { 02:00:01h. }\end{array}$ & $70.5 \%$ & $\begin{array}{l}\text { From } \\
\text { 22:00:01 to } \\
\text { 00:00 Until } \\
\text { 00: 00: 00h }\end{array}$ & $70.5 \%$ \\
\hline $\begin{array}{l}\text { From 02: 00: } \\
\text { 01h. Until 04: } \\
\text { 00: 01h }\end{array}$ & $41 \%$ & $\begin{array}{ll}\text { From } & \text { 00: } \\
\text { 00: } & \text { 00h } \\
\text { Until 04: 00: } \\
\text { 01h }\end{array}$ & $41 \%$ \\
\hline $\begin{array}{l}\text { From 04:01 } \\
\text { to 00:00. }\end{array}$ & $100 \%$ & $\begin{array}{l}\text { From 04:01 } \\
\text { to 00:00 }\end{array}$ & $100 \%$ \\
\hline
\end{tabular}

\section{RESULTS}

The microcontrollers, GPS and microchip is used to progress above discussed modules. A Universal Asynchronous Receiver Transmitter protocol which is used for communication provides the data transmission to a microprocessor employed in the system from the GPS. It provides one way of interaction and the waveform is shown in Figure 5.

A $I^{2} C$ communication bus is used to provide the communication between the RTC and the microcontroller. It provides two way communication between these 2 and the data is recorded in by the microcontroller using this bus. The resultant wave is shown in the $6^{\text {th }}$ Figure (Figure 6).

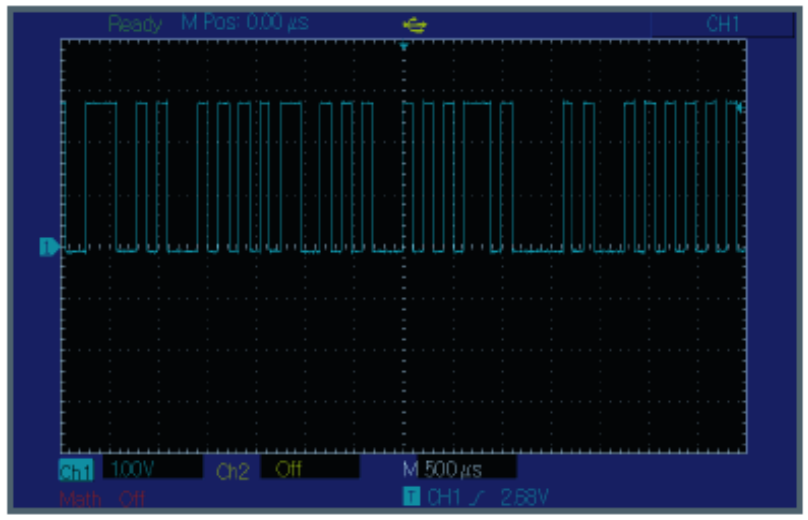

Fig. 5. The waveform of the signal of the UART protocol communication

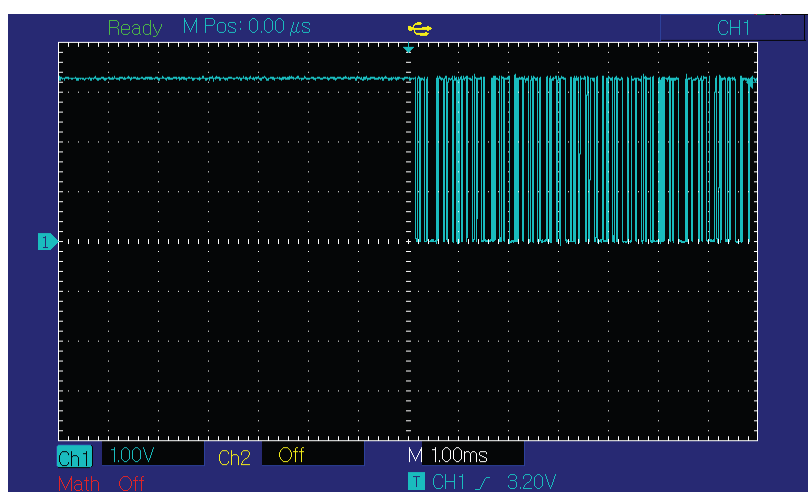

Figure 6 Communication between microcontroller and RTC

\section{CONCLUSION}

The projected work relates to an automatic street lighting system that does the operation of lighting on/off or dimming of light according to need automatically. The designed system is capable of doing the operation according to weather conditions and timing of a day with the help of microcontrollers, GPS and RTC. Two module related to the project have been discussed in section 2 in a simple way, Thus by checking the results obtained from the system, the system reduces the energy cost, cost of maintenance, increase in the life of lighting equipment. All the advantages are efficiently achieved by the system.

\section{REFERENCES}

1. Y. M. Jagadeesh, S. Akilesh, S. Karthik, and Prasanth, "Intelligent Street Lights," Procedia Technol., 2015.

2. Y. Wu, C. Shi, X. Zhang, and W. Yang, "Design of new intelligent street light control system," in 2010 8th IEEE International Conference on Control and Automation, ICCA 2010, 2010.

3. K. S. Sudhakar, A. A. Anil, K. C. Ashok, and S. S Bhaskar, "Automatic street Light Control System," Int. J. Emerg. Technol. Adv. Eng., 2010.

4. I. W. Pray et al., "GPS Tracking of Free-Ranging Pigs to Evaluate Ring Strategies for the Control of Cysticercosis/Taeniasis in Peru," PLoS Negl. Trop. Dis., 2016.

5. R. Husin et al., "Automatic street lighting system for energy efficiency based on low cost microcontroller," Int. J. Simul. Syst. Sci. Technol., 2012.

6. G. C, S. C, A. P. Kumar, K. A, and R. K.R, "Design and Implementation of Automatic Street Light Control System using Light Dependent Resistor," Int. J. Eng. Trends Technol., 2016.

7. J. Rigelsford, "GSM Networks: Protocols, Terminology and Implementation," Sens. Rev., 2014. 